\title{
ARTICLE OPEN \\ Machine learning guided appraisal and exploration of phase design for high entropy alloys
}

\author{
Ziqing Zhou ${ }^{1}$, Yeju Zhou ${ }^{2}$, Quanfeng $\mathrm{He}^{1}$, Zhaoyi Ding ${ }^{1}$, Fucheng Li ${ }^{1}$ and Yong Yang $\mathbb{D}^{1,3 *}$
}

High entropy alloys (HEAs) and compositionally complex alloys (CCAs) have recently attracted great research interest because of their remarkable mechanical and physical properties. Although many useful HEAs or CCAs were reported, the rules of phase design, if there are any, which could guide alloy screening are still an open issue. In this work, we made a critical appraisal of the existing design rules commonly used by the academic community with different machine learning (ML) algorithms. Based on the artificial neural network algorithm, we were able to derive and extract a sensitivity matrix from the ML modeling, which enabled the quantitative assessment of how to tune a design parameter for the formation of a certain phase, such as solid solution, intermetallic, or amorphous phase. Furthermore, we explored the use of an extended set of new design parameters, which had not been considered before, for phase design in HEAs or CCAs with the ML modeling. To verify our ML-guided design rule, we performed various experiments and designed a series of alloys out of the Fe-Cr-Ni-Zr-Cu system. The outcomes of our experiments agree reasonably well with our predictions, which suggests that the ML-based techniques could be a useful tool in the future design of HEAs or CCAs.

npj Computational Materials (2019)5:128; https://doi.org/10.1038/s41524-019-0265-1

\section{INTRODUCTION}

Since their advent in $2004{ }^{1}$ high entropy alloys (HEAs) have been attracting tremendous research interest because of their remarkable mechanical and physical properties. ${ }^{2-4}$ Compared with traditional alloys, HEAs usually contain more than five elements mixed with a similar atomic fraction, thereby also known as multiprincipal element alloys ${ }^{1}$ or compositionally concentrated alloys. ${ }^{2}$ Based on the assumption of ideal mixing, Yeh et al. ${ }^{5}$ proposed that an equal-atomic composition maximizes the configurational entropy of an HEA, therefore, in favor of the formation of random solid solution (SS) over other phases, such as intermetallics (IM). Conceptually, the idea of HEAs ${ }^{1}$ suggests that, as the number of the constituent elements increases in HEAs, the possibility for the formation of SS should increase. However, the formation of SS does not merely depend on the number of elements. According to the Hume-Rothery rules, ${ }^{6}$ the misfit in the physical and electronic properties of constituent elements strongly affects the formation of SS in binary alloys and possibly multicomponent alloys as well, such as HEAs.

Aside from SS, a variety of other phases, such as $\mathrm{IM}^{7,8}$ or even amorphous phase (AM), ${ }^{9-12}$ were observed in the as-cast HEAs. To rationalize this phenomenon, a number of empirical or semiempirical rules were proposed for phase selection in HEAs. These rules were developed mainly based on whether there was a correlation between the observed phases and several empirical parameters that could be calculated readily from a HEA composition, such as the ideal mixing entropy $S_{i d r}{ }^{1}$ the parameters of atomic size difference $\delta$ and the average mixing enthalpy $\Delta H_{\text {mix }}$ proposed by Zhang et al., ${ }^{8}$ the mean valance electron concentration (VEC) by Guo et al., ${ }^{13}$ the packing misfit parameter $\gamma$ by Wang et al., $^{14}$ the root mean square residual strain parameter by $\mathrm{Ye}$ et al., ${ }^{15}$ the electronegativity difference parameter by Guo et al., ${ }^{13}$ the dimensionless parameters $\Omega=\frac{T_{m} S_{i d}}{\left|\Delta H_{\text {mix }}\right|}$ ( $T_{m}=$ melting point) by Zhang et al. ${ }^{16}$ and $\phi=\frac{S_{i d}-\left|\Delta H_{\text {mix }}\right| / T_{m}}{\left|S_{E}\right|}\left(S_{E}=\right.$ excessive configuration entropy) by $\mathrm{Ye}$ et al. $^{17}$ and many others. ${ }^{18-22}$ Despite the seemingly difference among these rules, they are in the same spirit of the classic Hume-Rothery rule, i.e., aside from increasing the number of constituent elements, one has to minimize the difference in the physical and chemical properties of the constituent elements in HEAs in order to obtain random SS. On the other hand, alloying different types of elements can also facilitate the formation of metastable phases, such as $\mathrm{AM}^{23}$ or metastable random SS phases. ${ }^{24}$ Therefore, metastable phases have also been considered as one of the defining features of HEAs. ${ }^{18}$

At the fundamental level, phase selection in alloys, including metastable phases, can be attributed to the shape of their potential energy landscape (PEL), ${ }^{25-29}$ as illustrated in Fig. 1. In general, as PEL is derived from overall inter-atomic potentials, ${ }^{28}$ phase prediction in HEAs therefore must entail many-body interactions. On the other hand, phases in HEAs are commonly metastable in nature, ${ }^{30}$ which depend on the method of materials synthesis and processing. Because of these complexities, the issue of phase selection in HEAs is still open today although substantial efforts have been made over the past decade.

In this work, we would like to develop several machine learning $(\mathrm{ML})$ models to assess the widely accepted phase design rules for $\mathrm{HEAs}^{1,8,31,32}$ and also to explore new ones with additional design parameters, which have not been considered yet in the literature. To build a data set for the ML models, we include 601 different alloys and IM in the analyses. Because of the lack of data of thermal history, we only consider the compositions of as-cast alloys as the inputs to our ML models.

\footnotetext{
${ }^{1}$ Department of Mechanical Engineering, College of Engineering, City University of Hong Kong, Kowloon Tong, Kowloon, Hong Kong SAR, China. ${ }^{2}$ Department of Computer Science and Engineering, School of Engineering, The Hong Kong University of Science and Technology, Clear Water Bay, Kowloon, Hong Kong SAR, China. ${ }^{3}$ Department of Materials Science and Engineering, College of Engineering, City University of Hong Kong, Kowloon Tong, Kowloon, Hong Kong SAR, China. *email: yonyang@cityu.edu.hk
} 


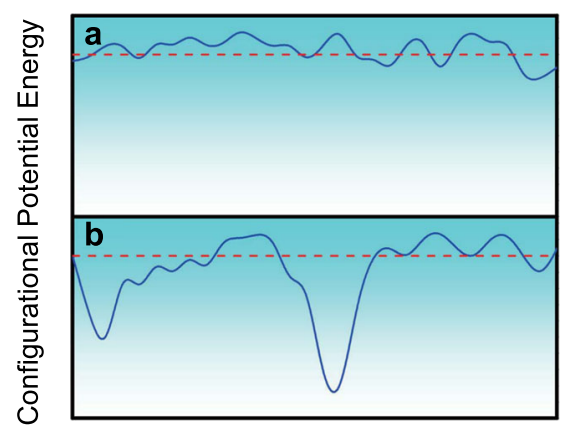

1D Configurational Coordinate

Fig. 1 The schematics of the one-dimensional potential energy landscape (PEL). The schematic PEL for a a high entropy phase and b a low entropy phase.

\begin{tabular}{|c|c|}
\hline Parameters & Formula \\
\hline Mean atom radius & $a=\sum_{i=1}^{n} c$ \\
\hline Atomic size difference & $\delta=\sqrt{\sum_{i=1}^{n} c_{i}\left(1-\frac{r_{i}}{a}\right)^{2}}$ \\
\hline $\begin{array}{l}\text { Average of the melting points of } \\
\text { constituent elements }\end{array}$ & $T_{m}=\sum_{i=1}^{r}$ \\
\hline $\begin{array}{l}\text { Standard deviation of melting } \\
\text { temperature }\end{array}$ & $\sigma_{T}=\sqrt{\sum_{i=1}^{n} c_{i}\left(1-\frac{T_{i}}{T_{m}}\right)^{2}}$ \\
\hline Average mixing enthalpy & $\Delta H_{\text {mix }}=4 \sum_{i \neq j} c_{i} c_{j} H_{i j}$ \\
\hline Standard deviation of mixing enthalpy & $\sigma_{\Delta H}=\sqrt{\sum_{i \neq j} c_{i} c_{j}\left(H_{i j}-\Delta H_{m i x}\right)^{2}}$ \\
\hline Ideal mixing entropy & $S_{i d}=-k_{B} \sum_{i=1}^{n} c_{i} \ln c_{i}$ \\
\hline Electronegativity & $X=\sum_{i=1}^{n} c_{i} X_{i}$ \\
\hline Standard deviation of electronegativity & $\Delta x=\sqrt{\sum_{i=1}^{n} c_{i}\left(x_{i}-x\right)^{2}}$ \\
\hline Average VEC & $V E C=\sum_{i=1}^{n} c_{i} V E C_{i}$ \\
\hline Standard deviation of VEC & $\sigma_{V E C}=\sqrt{\sum_{i=1}^{n} c_{i}\left(V E C_{i}-V E C\right)^{2}}$ \\
\hline Mean bulk modulus & $K=\sum_{i=1}^{n} c_{i} K_{i}$ \\
\hline Standard deviation of bulk modulus & $\sigma_{K}=\sqrt{\sum_{i=1}^{n} c_{i}\left(K_{i}-K\right)^{2}}$ \\
\hline
\end{tabular}

\section{RESULTS}

Our ML modeling is based on three algorithms, including the artificial neural network (ANN), the one-dimensional convolutional neural network (CNN), and the support vector machine (SVM), to assess the efficiency of the existing phase design rules and to explore new ones (see Methods). In principle, the three ML algorithms are suitable to solve supervised classification problems. ${ }^{33,34}$ ANN is based on a feed-forward structure with an input layer, one hidden layer as in our work and an output layer. ${ }^{33}$ ANN has recently been used preferably in material informatics because of its good performance on small data sets. ${ }^{34-36} \mathrm{CNN}$ is based on a deep neural network and attached to convolutional and pooling layers. ${ }^{33}$ By comparison, it has the most complicated architecture and runs slow. Simply speaking, SVM is an algorithm to search hyperplanes through data division. ${ }^{37}$ It has the simplest architecture and runs fast; however, the outcome of SVM is highly sensitive to the choice of kernel parameters.

In the literature, ${ }^{1,8,31,32}$ there are already a few widely accepted parameters for the general phase design in HEAs, including the atomic size difference $(\delta)_{1}^{8}$ the mixing enthalpy $\left(\Delta H_{\text {mix }}\right)_{1}^{8}$ the ideal mixing configurational entropy $\left(S_{i d}\right){ }^{38}$ and the standard deviation of electronegativity $(\Delta x))^{32}$ From a statistic mechanics viewpoint, those parameters can be related to the PEL of an alloy. ${ }^{26}$ However, for a more comprehensive exploration, we would like to expand the parameter space by adding more thermodynamic parameters, which were not considered previously. In principle, for each parameter considered, we calculate both its mean value and standard deviation for a multicomponent alloy wherever is possible. As a result, this leads to 13 parameters (shown in Table 1).

For the ML modeling, these parameters were normalized based on the general expression $x_{i}=\frac{x_{i}^{\text {(old })}-x_{i m i n}^{\text {(old })}}{x_{i \max }^{(\text {old })}-x_{i \min }^{\text {(old }}},(i=1,2, \ldots, 13)$, where $x_{i}^{(\text {old })}$ and $x_{i}$ denote, respectively, the original and normalized value of the ith parameter or the ith feature of our ML model. To be consistent with the HEA literature, ${ }^{2,8,39}$ we consider the existence of three general phases, i.e., random SS, IM, and AM, as the outputs or the data labels of our ML modeling. To be specific, the existence for the individual phase is quantified by either 0 (not existent) or 1 (existent). The label value of our ML model is a binary number, which indicates whether a given phase can be formed. If yes, the label value is 1 ; if no, it is 0 .

To train our ML model, we collected the data from 163 binary alloys, 120 ternary alloys, 89 quaternary alloys, and 229 higher order alloys with at least five elements. Totally, we built a data set containing 601 alloys, including 165 pure AM alloys, 248 pure intermtallics, 131 pure random SS, 6 mixture of AM and intermetallic alloys, and 51 mixture of intermetallic and random SS alloys (see Supplementary Table 5). Following the ML literature, ${ }^{40}$ the data set was randomly divided into three subsets, with $70 \%$ data for training, $15 \%$ for validation and $15 \%$ for testing. After data training, the ANN model achieved a high testing accuracy of $98.9 \%, 97.8 \%$, and $95.6 \%$ for AM, SS, and IM, respectively; the 1D CNN model achieved a similar testing accuracy of $97.8 \%$ for AM, $98.9 \%$ for SS, and $94.4 \%$ for IM; whereas the SVM model achieved the testing accuracy of $96.7 \%$ for AM, $98.9 \%$ for SS, and $95.6 \%$ for IM. Furthermore, we tested the robustness of our algorithms by performing 10 times of independent data division and model training. As seen in Supplementary Table 1, the testing accuracies of our ML models based on the 10 independent data divisions are very similar, suggesting that the outcome of our ML modeling is not sensitive to data division.

For the phase design of HEAs, it is of great importance to know how the properties or attributes of the constituent elements influence the formation of a particular phase. Theoretically, this could translate to the measure of sensitivity of the data label, i.e., the probability to form a certain phase, with respective to changes in the corresponding features, i.e., thermodynamic or micromechanical parameters, as designed for our ML models. Through the compound transformation function, the parametric sensitivity of the data labels can be assessed quantitatively, which connects the features and labels through the well-trained ML model. For simplicity, let us focus on the ANN model for which an analytic expression for the compound transformation function is available. For our ANN model, the compound transformation function between the features $\mathbf{X}$ and the labels $\boldsymbol{z}$ is highly nonlinear, which 
can be expressed as:

$z=\mathbf{W}_{2} \cdot \frac{1}{1+e^{-\mathbf{W}_{1} \cdot \mathbf{x}+\mathbf{b}_{1}}}+b_{2}$

$\mathbf{W}_{1}$ is the linear transformation matrix between the input layer and the hidden layer; $\mathbf{W}_{2}$ is the linear transformation matrix between the hidden layer and the output layer; $\mathbf{b}_{1}$ and $b_{2}$ denote the biases. With the Taylor's expansion $\frac{1}{1+\mathrm{e}^{-x}}=\frac{1}{2}+\frac{1}{4} x+o\left(x^{2}\right)$, Eq. (1) can be linearized as follows:

$z=\frac{1}{4} \mathbf{W}_{2} \cdot \mathbf{W}_{1} \cdot \mathbf{X}+\frac{1}{4} \mathbf{W}_{2} \cdot\left(\mathbf{b}_{1}+\mathbf{2}\right)+b_{2}$

From Eq. (2), we can obtain a simple scaling relation $z \sim \mathbf{W}_{2} \cdot \mathbf{W}_{1} \cdot \mathbf{X}$, based on which we define the sensitivity matrix as $\mathbf{S}=\mathbf{W}_{2} \cdot \mathbf{W}_{1}$. Note that, for the sensitivity matrix $\mathbf{S}$, the number of rows correspond to the number of phases, whereas the number of columns correspond to the number of features.

With the well-trained ANN models, we obtained the sensitivity matrices for the three phases (See Supplementary Tables 2-4). The entries to each sensitivity matrix could be understood as the measure of influence of the individual design parameter on the formation of a certain phase or the correlation between the design parameter and phase. In concept, the larger is the magnitude of a sensitive parameter, the more influential is the corresponding design parameter and vice versa. Note that a sensitivity measure could be negative for a negative correlation, or positive for a positive correlation. To assess the influence of the parameters, we evaluated the magnitude of the sensitivity value of each feature, from which one can infer that features with a low magnitude of sensitivity might be abandoned or disposed of. These disposable features were statistically unimportant and can be considered as redundant. After removing the redundant features, we re-trained the ANN model and found that its testing accuracy could be somewhat improved; however, over-reduction of the features caused some loss of testing accuracy of the ML model. For example, as for the ANN model, we determined that nine features are essential for the AM phase, 11 features for the IM phase, and 10 features for the SS phase. Table 2 compares the performance of the ML models before and after the feature reduction. Statistically, it appears that 13 features work the best for the CNN model, which has the most complicated topologic structure and may need more training variables for a high accuracy. By comparison, features slightly fewer than 13 in number do not affect the accuracy obviously for the ANN and SVM models. In general, inclusion of all the 13 features does not significantly sacrifice the testing accuracy of our ML models, as seen in Table 2.

\section{DISCUSSION}

In order to obtain statistically reliable sensitivity measures, we trained each ANN model for 30 times and extracted the sensitivity matrices accordingly. After that, the values of each sensitivity measure were averaged with the standard deviation being computed. Figure 2 shows the comparison of the sensitivity measures which are color coded based on the ratio of mean to standard deviation. If the averaged sensitivity measure is positive

Table 2. Comparison of the testing accuracy of the ANN, CNN, and SVM models before and after feature reduction.

\begin{tabular}{llllllll}
\hline & \multicolumn{3}{c}{ Original } & 13 features & & & \multicolumn{2}{l}{ Reduced features } \\
\cline { 2 - 3 } & ANN & CNN & SVM & & ANN & CNN & SVM \\
\hline AM & $98.9 \%$ & $96.7 \%$ & $97.8 \%$ & & $96.7 \%$ & $93.3 \%$ & $94.4 \%$ \\
IM & $95.6 \%$ & $95.6 \%$ & $94.4 \%$ & & $94.4 \%$ & $92.2 \%$ & $94.4 \%$ \\
SS & $97.8 \%$ & $98.9 \%$ & $98.9 \%$ & & $98.9 \%$ & $97.8 \%$ & $98.9 \%$ \\
\hline
\end{tabular}

for a positive correlation, it would be colored in red; if the averaged sensitivity measure is negative for a negative correlation, it would be colored in blue. Depending on the ratio of mean to standard deviation, the intensity of color varies. In Yeh's original work, ${ }^{1} S_{i d}$ was taken as the defining parameter for the formation of SS in HEAs. The higher is $S_{i d}$ the more likely is to form SS over other phases. This manifests as a high positive sensitivity measure $(+13)$ of $S_{i d}$ for SS. By comparison, the sensitivity measure of $S_{i d}$ for IM is negative $(-7)$, which somewhat corroborates the notion of the high entropy effect proposed by Yeh. ${ }^{1}$ Interestingly, the sensitivity measure of $S_{i d}$ for AM is positive but with a moderate magnitude $(+5)$. This behavior suggests that increasing the chemical complexity does promote glass formation, which is in line with one of the well-known design rules proposed by Inoue. ${ }^{31}$

Based on the sensitivity measures (Fig. 2), it is clear that $\delta$ plays a very important role on the formation of SS, whereas $\Delta H_{\text {mix }}$ affects the formation of SS almost to the same degree as $S_{\text {id }}$. Evidently, the very negative sensitivity measure $(-16)$ implies that one has to minimize the atomic size mismatch in order to obtain SS. Conversely, the positive sensitivity measure suggests that one should increase the atomic size mismatch in order to obtain IM or AM. This agrees with a number of well-established empirical rules, such as the Hume-Rothery rule, ${ }^{41}$ one of the Inoue's rules ${ }^{31}$ and the very recent rule proposed by Zhang et al. ${ }^{8}$ Now let us focus on the design parameter $\Delta H_{\text {mix }}$. As the $\Delta H_{\text {mix }}$ values are mostly negative for different atomic pairs, therefore, if one would like to reduce the magnitude of $\Delta H_{\text {mix }}$ of an alloy, he had to increase $\Delta H_{\text {mix }}$ of the alloy such that it would get closer to zero. This explains why the sensitivity measure $(+11)$ of SS on $\Delta H_{\text {mix }}$ is positive because a near-zero value of $\Delta H_{\text {mix }}$ is beneficial to SS according to Zhang et al. ${ }^{8}$ In contrast, a more negative $\Delta H_{\text {mix }}$ is in favor of IM and AM, as discussed in previous work. ${ }^{8,42}$ As shown in Fig. 2, the sensitivity measures of $\delta$ and $\Delta H_{\text {mix }}$ for SS are larger than those for IM and AM. As seen in Fig. 3, SS has a rather narrow distribution of $\delta$ and $\Delta H_{\text {mix }}$ in comparison with $\mathrm{AM}$ and IM. This indicates that the formation of SS is more sensitive to the change in $\delta$ or $\Delta H_{\text {mix }}$ than that of IM and $\mathrm{AM}$, which agrees with our sensitivity measure and also the recent works. ${ }^{8,20}$ In the prior work, ${ }^{32}$ increasing the value of $\Delta x$ was thought to promote the formation of IM while suppress that of SS. This is also in line with the sensitivity measure of $\Delta x$ for IM and SS. The negative sensitivity measure of $\Delta x$ for $A M$ suggests that one has to reduce $\Delta x$ for the formation of $A M$. In general, the reduction in $\Delta X$ benefits both IM and $A M$ and this behavior is very different from that of $\Delta H_{\text {mix }}$.

For a quantitative comparison, we re-built our ML models with the four conventional features aligned with the classic phase design rules, ${ }^{1,6,8,31,32}$ including $S_{i d}, \delta, \Delta H_{\text {mix }}$ and $\Delta x$ (see Methods). After that, these ML models were trained and the test accuracies are shown in Table 3. Evidently, the ML model based solely on the single parameter $S_{i d}$ has the poorest performance in terms of the testing accuracy, especially for IM ( 62\%). In general, the addition of other design parameters, such as $\delta, \Delta H_{\text {mix }}$, and $\Delta x$, improves the accuracy for all three phases. By comparison, it is clear that the ML model based on the full set of the design parameters outperforms other ML models based on a partial set of the design parameters.

Next, let us move to the new design parameters that were not considered for alloy screening and phase design in the prior works, as shown in Fig. 2. Note that VEC was early proposed by Guo et al. ${ }^{13}$ to distinguish different crystalline structures of a SS phase (FCC versus BCC); however, it was seldom used for phase selection among SS, IM, and AM. Therefore, we herein consider VEC as a new design parameter in our ML modeling. To understand the effect of these new design parameters, we resort to the potential energy fluctuation (PEF) model we recently developed. ${ }^{26}$ As illustrated in Fig. 1, the alloy system with a smooth potential energy profile has a high configurational entropy and tends to form SS; whereas the alloy system with a 


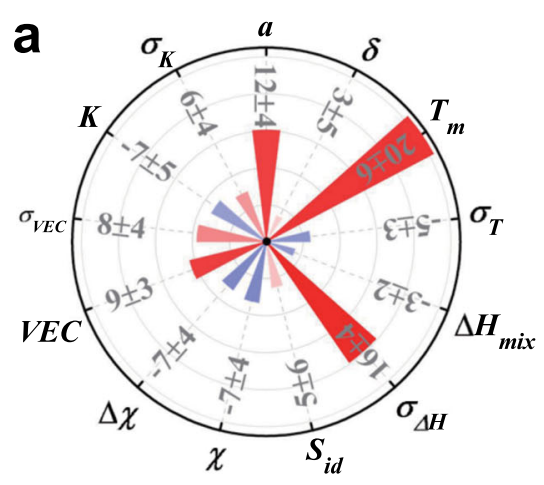

$\mathbf{A M}$

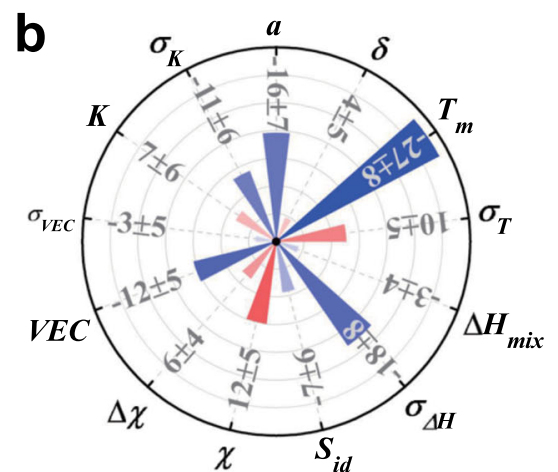

IM
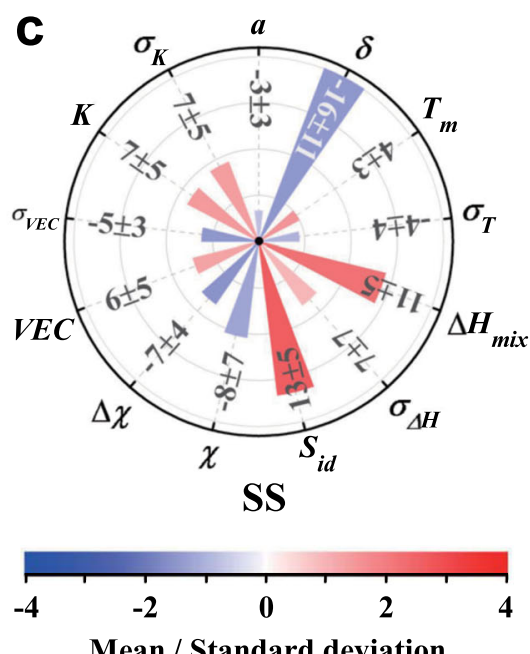

Fig. 2 Comparison of the sensitivity measures of the 13 design parameters based on the result of the ANN model. The sensitivity measures of the 13 design parameters for (a) amorphous phase (AM), (b) intermetallics (IM) and (c) solid solution (SS).

Table 3. Comparison of the accuracy of 1-feature $\left(S_{i d}\right)$ model, 3-feature $\left(S_{i d}, \delta, \Delta H_{\text {mix }}\right)$ model, 4-feature $\left(S_{i d}, \delta, \Delta H_{\text {mix }}, \Delta X\right)$ model, and 13feature model.

\begin{tabular}{lllll}
\hline & $S_{i d}$ & $S_{i d} \delta, \Delta H_{\text {mix }}$ & $S_{i d} \delta, \Delta H_{\text {mix }}, \Delta x$ & 13 features \\
\hline AM & $72.2 \%$ & $85.6 \%$ & $87.8 \%$ & $95.6 \%$ \\
IM & $62.2 \%$ & $75.6 \%$ & $83.3 \%$ & $92.2 \%$ \\
SS & $83.3 \%$ & $92.2 \%$ & $94.4 \%$ & $97.8 \%$ \\
\hline
\end{tabular}

rugged potential energy has a low configurational entropy and tends to form IM or even metastable phases, such as AM. According to the PEF model, ${ }^{25}$ the PEF brought about by the chemical and mechanical interaction among constituent elements can be quantified as follows:

$x_{e}=4.12 \delta \cdot \sqrt{\frac{K V}{k_{B} T}}$

$x_{c}=2 \sqrt{\frac{\sigma_{\Delta H}}{k_{B} T}}$

where $x_{e}$ stands for the extended atomic size difference; $x_{c}$ stands for the chemical bond misfit; $K$ is the bulk modulus; $V \sim a^{3}$ is the mean atomic volume; $k_{B}$ is the Boltzmann constant, and $T$ is the temperature of the system or the melting temperature at which a liquid solidifies into a solid in arc melting. In order to obtain SS, one has to minimize both $x_{e}$ and $x_{c}$. In theory, it will facilitate the

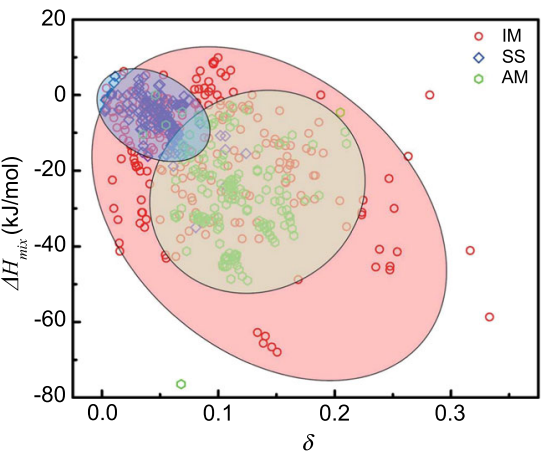

Fig. 3 The possible correlation of the design parameters and experimentally obtained phases. The plot of $\delta$ vs $\Delta H_{\text {mix }}$ for SS, IM, and $A M$.

formation of SS if one could reduce $\delta, K, a$, and $\sigma_{\Delta H}$, whereas simultaneously increase the melting point $T$.

Now let us discuss the effect of $T_{m}$. Here, we should first emphasize $T_{m}$ is the average of the melting points of constitutive elements rather than the real melting point or the liquidus temperature of an alloy. From Fig. 2, one can infer that a high $T_{m}$ value favors AM while a low $T_{m}$ value favors IM. From a statistical viewpoint, this suggests that, in the past, one tended to select high melting point elements for $\mathrm{AM}$, whereas low melting point elements for IM. According to Lu et al., ${ }^{43}$ the glass transition point $T_{g}$ of a metallic glass is correlated with $T_{m}$ through the relation 
$T_{g}=0.385 T_{m}$. Therefore, the higher is $T_{m}$ the higher is $T_{g}$. As the glass forming ability of metallic glasses increases with the reduced temperature $T_{g} / T_{l}{ }^{44}$ where $T_{l}$ stands for the alloy's liquidus temperature, a higher $T_{m}$ value and hence a higher $T_{g}$ value indicates a better glass forming ability, which is consistent with our ML result. In addition, it is worth mentioning that $a, T_{m}$ and $K$ are not statistically independent. As shown in Fig. 4, $a$ and $K$ are negatively correlated, whereas $T_{m}$ and $K$ are positively correlated. This inter-dependence explains the effect of $a, T_{m}$ and $K$, as seen in Fig. 2.

Based on our findings, it appears that both high $K$ and high $\sigma_{K}$ are beneficial for SS, high $K$ and low $\sigma_{K}$ for IM, whereas low $K$ and high $\sigma_{K}$ for AM. Although VEC was mainly used to distinguish different SS phases in the previous work, ${ }^{17}$ however, our model shows that VEC and $\sigma_{V E C}$ as well as $X$ also participates in phase selection. As seen in Fig. 2, high VEC, low $\sigma_{V E C}$, and low $X$ are in favor of SS; low VEC, low $\sigma_{V E C}$ and high $X$ are in favor of IM, whereas high VEC, high $\sigma_{V E C}$, and low $X$ are in favor of AM. This intriguing behavior indicates that VEC should play a role in phase formation. According to the hybridization theory proposed by Fukuhara et al., ${ }^{45} \mathrm{AM}$ alloys tend to have larger VEC than crystalline metals. This is because the probability of forming metallic glass increases with more valence electrons, such as $s, p$, and even $d$ or $f$ electrons, owing to the formation of a pseudo gap below the Fermi energy $E_{F}$ by spd or spf hybridization. ${ }^{46}$ Although the definition of VEC used by Fukuhara et al. differs from that in
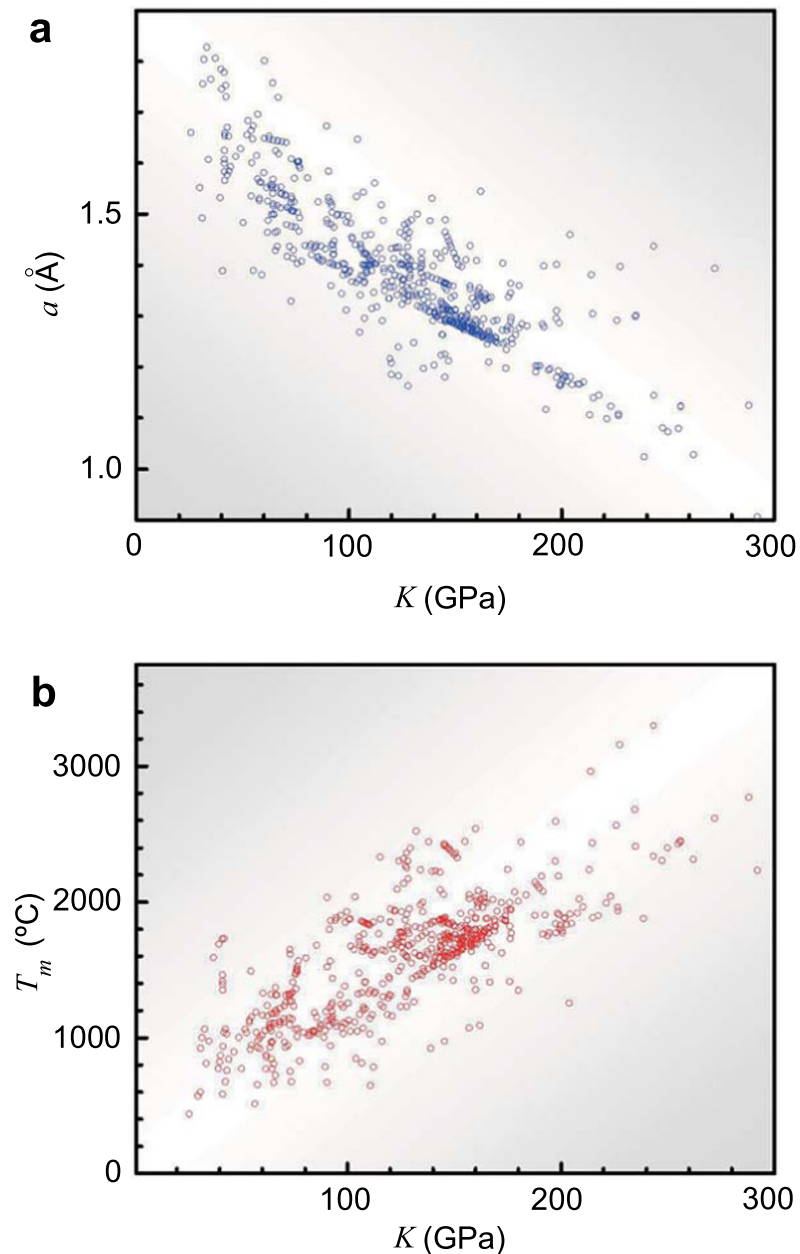

Fig. 4 The statistical correlations between the properties of the constituent elements. The correlations between a atom size and bulk modulus and between $\mathbf{b}$ melting temperature and bulk modulus. (See Supplementary Table 5 for the details of the data). our work, however, the VEC values calculated with the two different definitions for the alloys are positively correlated (see supplementary Fig. 1). That behavior suggests that the VEC-related mechanisms proposed by Fukuhara et al. ${ }^{45}$ could be applicable to our case as well. In addition, our ML model also indicates that low $\sigma_{T}$ favors SS and AM, whereas high $\sigma_{T}$ favors IM.

To further validate our ML modeling, we carried out extensive experiments through the development of a new hybrid HEA system, which has a general chemical composition of $(\mathrm{FeCrNi})_{10-x}$ $(\mathrm{ZrCu})_{\mathrm{x}}(x=1,2, \ldots, 8)$, where $x$ stands for the atomic percentage. First, we obtained a series of the bulk HEAs through arc melting (see Methods). As shown in Fig. 5a, the XRD results show the phases detected in the HEA bulk samples, being labeled as S1 to S8, with the corresponding $x$ value increasing from 1 to 8 . Evidently, a dual phase SS (FCC + BCC) structure is observed for $x=1$ and 2 . Interestingly, when $x$ further increases to $3-5$, both SS and IM phases can be detected. Finally, when $x$ reaches a relatively high value of $6-8$, only IM phases can be detected in the HEA. Figure $5 \mathrm{~b}$ shows the atomic fraction of the constituent elements in the as-cast HEA samples, based on which we can predict phase selection in each of the HEA samples with our ML models. As seen in Fig. $5 c$, the phase in the bulk sample is predicted to transit from SS, to SS + IM (multi-phase), IM + AM (multi-phase) and finally to AM. The prediction of SS and IM in $x=1-3$ agrees with our experimental results. Nevertheless, our ML modeling predicts that the HEAs should transit to IM + AM and AM when $x$ increases, which seemingly contradicts our experimental results. As discussed in the later text, this discrepancy could be attributed to a cooling rate effect.

To study the cooling rate effect, we prepared a few ribbon samples through vacuum melt spinning (see Methods), which had the compositions of $(\mathrm{FeCrNi})_{2}(\mathrm{ZrCu})_{8}(\mathrm{~S} 8),(\mathrm{FeCrNi})_{1.5}(\mathrm{ZrCu})_{8.5}(\mathrm{~S} 9)$, and $(\mathrm{FeCrNi})_{1}(\mathrm{ZrCu})_{9}(\mathrm{~S} 10)$. As shown in Fig. $6 \mathrm{a}$, it is evident that the atomic structure of the ribbon samples transits from $\mathrm{SS}+\mathrm{AM}$ to $\mathrm{AM}$ with the increasing concentration of $\mathrm{ZrCu}$, which agrees well with the prediction of our ML modeling. Furthermore, we prepared a series of thin films through co-sputtering (see Methods). Figure $6 c$ shows the XRD curves obtained from FeCrNi-rich compositions to ZrCu-rich compositions. Evidently, as the chemical composition continuously changes from FeCrNi to $\mathrm{ZrCu}$, the HEA films undergo a transition from a crystalline to AM structure. This behavior generally agrees with the prediction of our $\mathrm{ML}$ modeling (Fig. 5c). Figure $6 \mathrm{~b}$ shows the exact composition of the thin-film samples we obtained. According to Fig. $5 c$, these compositions should fall into the region where IM transitions to AM. In other words, even if the atomic structure with these compositions became AM under a very high cooling rate, as is the case of thin-film deposition, one would expect an increasing glass forming tendency with the increasing atomic fraction of the $\mathrm{ZrCu}$ component in our HEA, which warrants future research.

To summarize, we developed the $\mathrm{ML}$ models based on three algorithms (ANN, CNN, and SVM) to assess the existing phase design rules for HEAs in this work. With these ML models, we further explored the use of additional new design parameters for phase design for HEAs. In theory, these new design parameters can be associated with the fluctuation of PEL of a multicomponent system, which greatly improves the accuracy of our ML modeling. To verify the ML modeling, we carried out a series of experiments, including casting, melt spinning, and co-sputtering, for the design of a new Fe-Cr-Ni-Zr-Cu HEA system. The experimental results generally agree with the prediction of our ML modeling. However, in the meantime, our results also indicate clearly that the phases in the HEAs designed based on our ML modeling are cooling rate dependent, which is sensible as AM is a metastable phase and even SS in some HEAs, such as the cantor alloy, ${ }^{7}$ are also metastable in nature. 

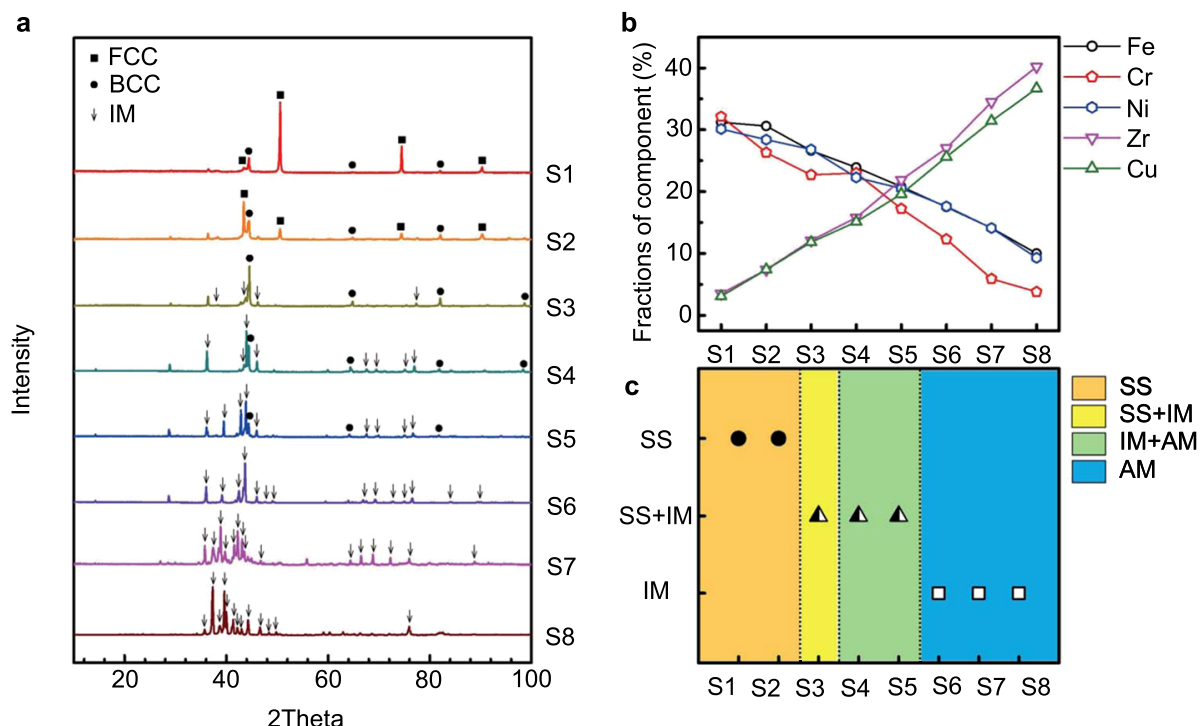

Fig. 5 The structural characterization of the bulk $(\mathrm{FeCrNi})_{10-x}(\mathrm{ZrCu})_{\mathbf{x}}(\mathbf{x}=1, \mathbf{2}, \ldots, \mathbf{8})$ alloy. a The compiled XRD results, $\mathbf{b}$ the compiled EDX results, and $\mathbf{c}$ the comparison of the phases obtained for different alloy compositions from arc melting (SS: filled circle, SS + IM: half-filled triangle, and IM: hollow square) against the ML predictions. Note that the colors manifest the ML predictions (SS: orange, SS + IM: yellow, IM + AM: green, and AM: blue).
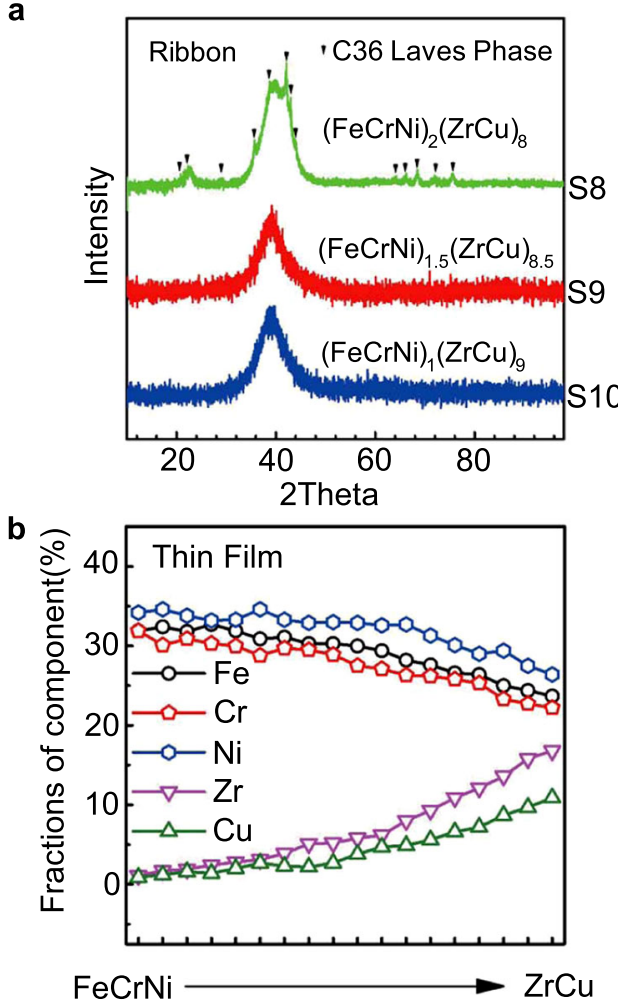

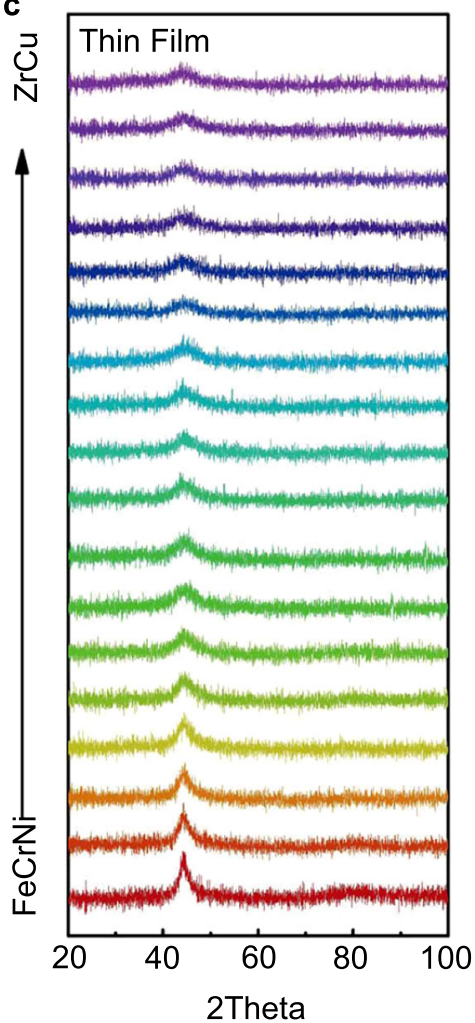

Fig. 6 The structural characterization of the Fe-Cr-Ni-Zr-Cu ribbons and thin films. a The XRD spectra for the melt spinning ribbons, $\mathbf{b}$ the compositions of the thin films detected via EDX, and $\mathbf{c}$ the XRD spectra for the thin films.

\section{METHODS}

$\mathrm{ML}$ algorithms

ANN. As illustrated in Fig. 7, our first ML model is based on a classification ANN with one hidden layer. The neural network contains one input layer for the 13 design parameters, one hidden layer with 20 neurons and one output layer for the label. The transform function that connects the input and hidden layer can be written as $\mathbf{a}=\mathbf{W}_{1} \mathbf{x}+\mathbf{b}_{1}$, where $\mathbf{a}_{(20 \times 1)}$ is the vector composed of the values received by the 20 hidden neurons; $\mathbf{W}_{\mathbf{1}(20 \times 13)}$ is the weight matrix derived from linear transformation, $\mathbf{x}_{(13 \times 1)}$ is the normalized feature of our $\mathrm{ML}$ model and $\mathbf{b}_{\mathbf{1}(20 \times 1)}$ is the offset of the linear transformation. Furthermore, the sigmoid function $S(x)=\frac{1}{1+e^{-x}}$ was used as the activation function in the hidden layer. Similarly, the transform function between the hidden and output 


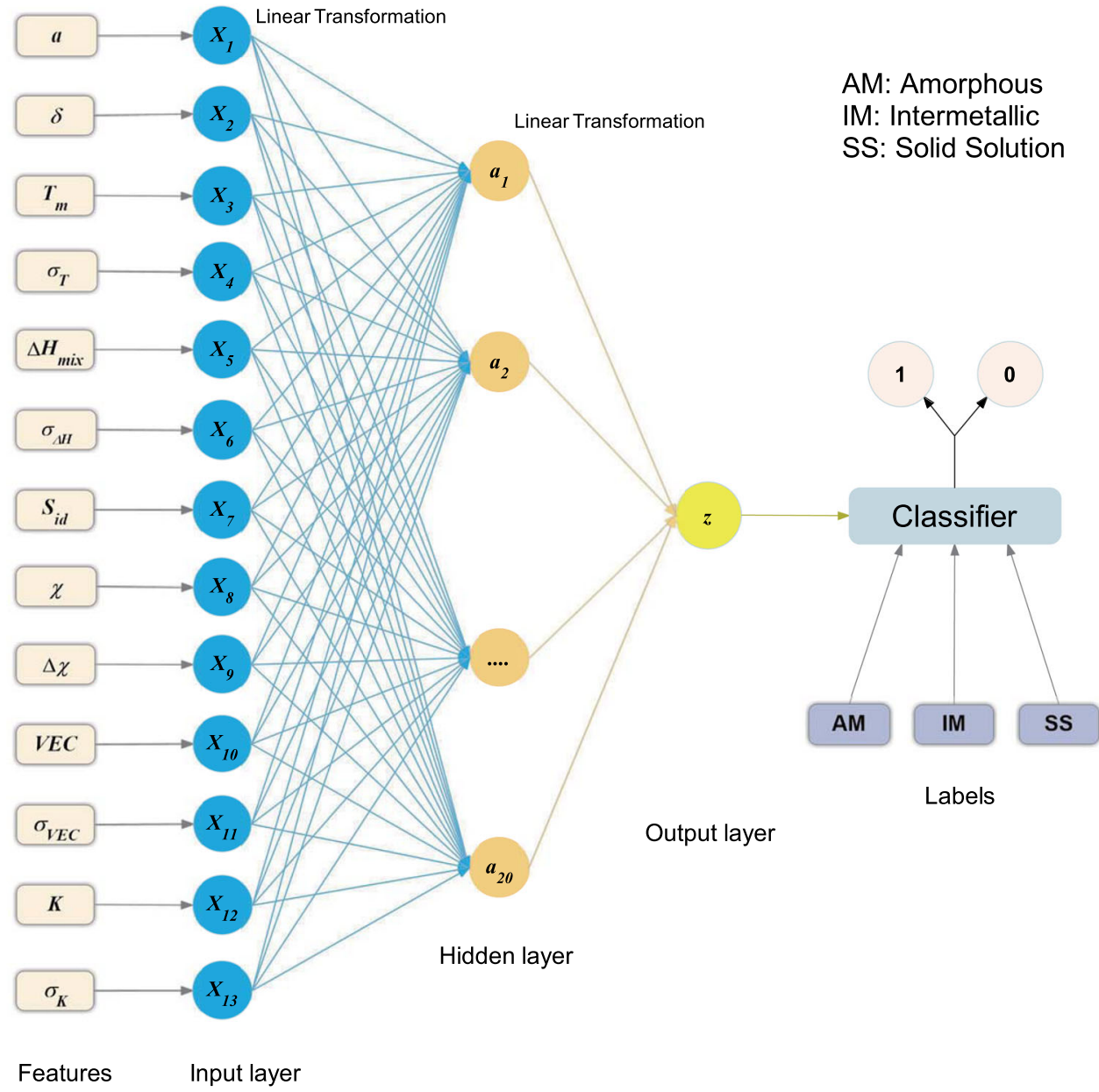

Fig. 7 The architecture of the machine learning model. The schematic for the design of the artificial neural network-based machine learning model.

layer can be written as $z=W_{2} h+b_{2}$, where $\mathbf{W}_{\mathbf{2}(1 \times 20)}$ is the weight matrix of the linear transformation, $\mathbf{h}_{(20 \times 1)}$ is the output of the hidden layer, and $b_{2}$ is the offset of the linear transformation. Here, it is worth mentioning that we used cross-entropy as the loss function in our ANN model, which was defined as $\mathrm{H}=-\sum_{i=1}^{n}$ ylogy, where variable $y$ is the label of the training data and $\hat{y}=z$ is the model output. In general, cross-entropy measures the difference between the real label (or real data) and model output, which can be treated as the systematic error of our ML model and needs to be minimized during a data training process. After the model training, $\mathbf{W}_{1}, \mathbf{W}_{2}, \mathbf{b}_{1}$, and $b_{2}$ are obtained. Afterwards, the well-trained models can be used for predictions through the transition functionz $=W_{2} \frac{1}{1+e^{-W_{1} X+b_{1}}}+b_{2}$, where $\mathbf{X}$ stands for the testing subset. In the classifier, the value $z$ would be rounded up to 1 or 0 , which corresponds to the existence or absence of a certain phase, respectively.

One-dimensional CNN. Figure 8 illustrates the topological structure of our second ML model, which is the one-dimensional CNN with two hidden layers. The Kernel size and stride are set to 3 and 1 for both convolutional layers. LeakyReLU is used as the activation function for the two hidden layers. For an input $u$, LeakyReLU $(u)=u$ if $u>0$ and $0.01 u$ if $u<0$. The input layer contains 13 features, which are the same as those for the aforementioned ANN model. The first hidden layer consists of 64 channels, each of which contains 11 neurons. Each neuron takes weighted values from only three inputs in proximity. For instance, $u_{1}=\operatorname{LeakyReLU}\left(W_{1}^{u}\left(x_{1}+\right.\right.$ $\left.\left.x_{2}+x_{3}\right)+b_{1}^{u}\right)(W$ denotes weight, $b$ denotes bias). Sixty-four channels are stacked together as the first hidden layer (convolutional layer) with different weights and biases in every of the 11 neurons. The second hidden layer consists of 128 channels, each containing nine neurons. Each neuron takes weighted values from three inputs in proximity. The 128 channels, which contain different weights and biases for each of the nine neurons, are stacked together as the second hidden layer (convolutional layer). The output layer unstacks the 128 channels of second hidden layer neurons, and then calculates two weighted sums $\left(z_{1}, z_{2}\right)$ as scores for the classification with a formula of $z_{j}=W_{1 j}^{z} v_{1}+W_{2 j}^{z} v_{2}+W_{3 j}^{z} v_{3}+\cdots+$ $W_{1152 j}^{z} v_{1152}$ (for $j=1,2$ ). The scores are normalized via $\sigma\left(z_{j}\right)=\frac{e_{j}^{z}}{\sum_{k=1}^{2} e^{z_{k}}}$ (for $j=1,2$ ) where $\sum_{i=1}^{2} \sigma(\mathbf{z})_{i}=1$ and $\sigma(\mathbf{z})_{i} \in(0,1)$. The cross-entropy loss function was defined as $H=-\sum_{i=1}^{n} \mathbf{y} \log \hat{\gamma}^{\top}$, where vector is the label of the training data and $\hat{y}_{(1 \times 2)}=\left(\sigma_{1}, \sigma_{2}\right)$ is the model output. Convolutional layers extract spatial correlations between certain features and achieve the high and stable accuracy at cost of much more trainable variables.

SVM. SVM with a radial basis function kernel is applied as the third ML model. The kernel function is $k\left(\mathbf{x}, \mathbf{x}^{\prime}\right)=\exp \left(-\gamma\left\|\mathbf{x}-\mathbf{x}^{\prime}\right\|^{2}\right)$ where $\gamma=$ $0.4,0.2,0.2$ for SS, AM, and IM, respectively. The regularization parameter $C$ is set to 10 for all three classes. Because there is a tradeoff between the number of points misclassified and the robustness of the classification among new data, we tuned the parameters to reach the highest classification accuracy.

The ML model with one, three, and four features

To build the ML models with a partial set of the design parameters, we utilized the ANN algorithm, as shown in Fig. 8. To be specific, the feature in the one-feature model is $S_{i d i}$ the features in the three-feature model comprise $S_{i d}, \delta$, and $\Delta H_{\text {mixi }}$ whereas the features in the four-feature model comprise $S_{i d} \delta, \Delta H_{m i x}$ and $\Delta X$.

Casting of bulk samples through Arc melting. We used pure metals with a purity level higher than $99.9 \%$ to prepare the bulk samples $(\mathrm{FeCrNi})_{10-x}$ $(\mathrm{ZrCu})_{x}(x=1,2, \ldots, 8)$. The raw elemental materials, including $\mathrm{Fe}, \mathrm{Cr}, \mathrm{Ni}, \mathrm{Zr}$, 


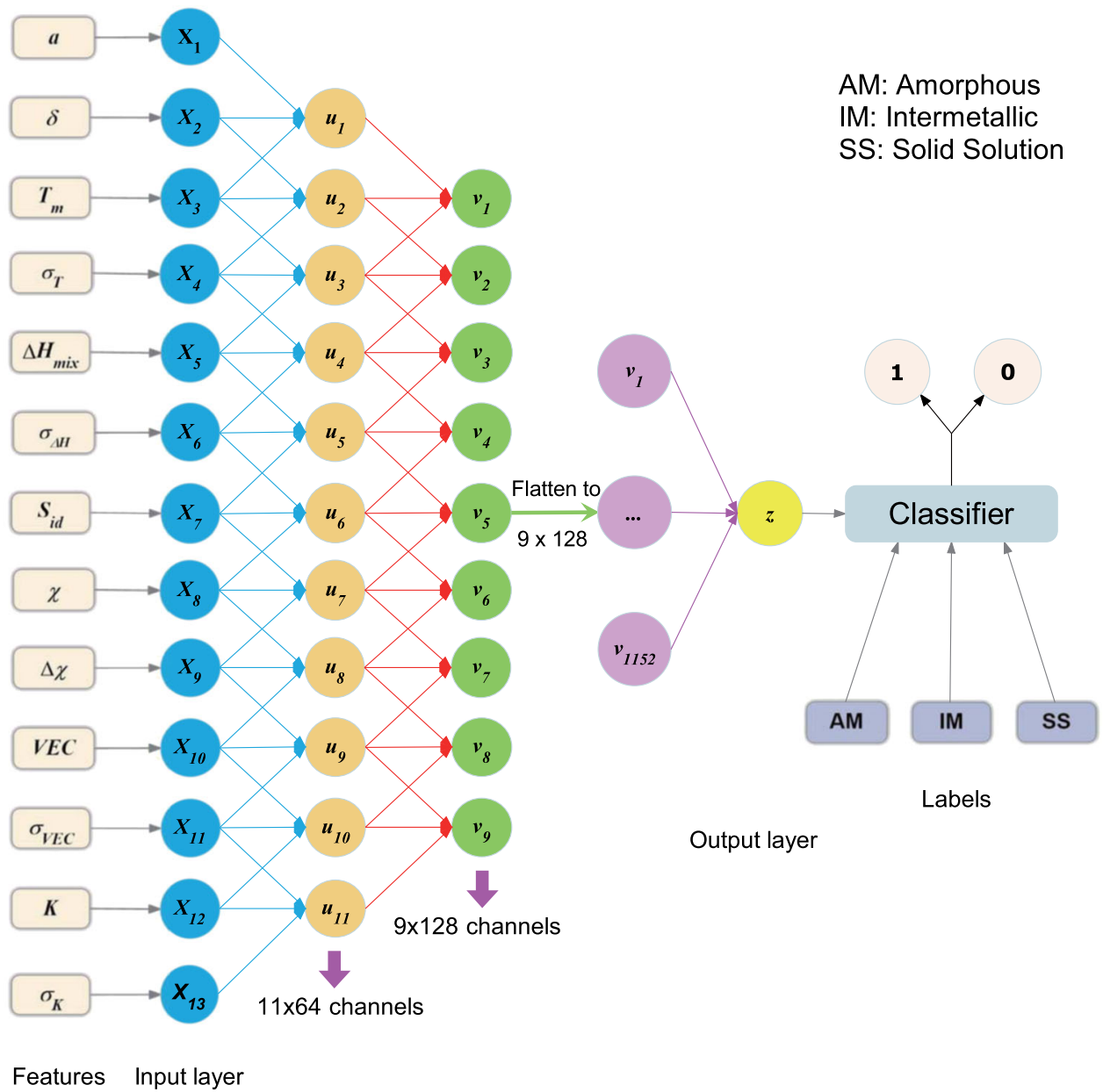

Fig. 8 The architecture of the machine learning model. The schematic of one-dimensional convolutional neural network-based machine learning model.

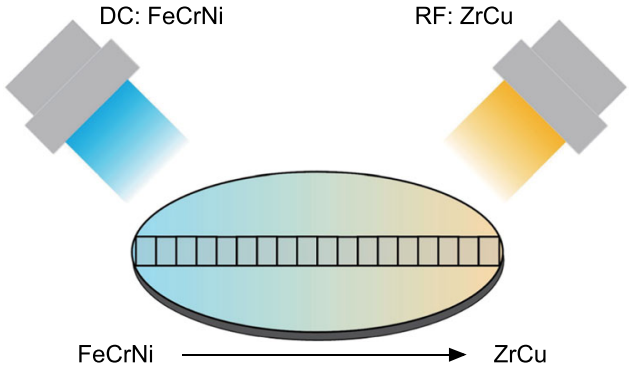

Fig. 9 The design of the high-throughput experiments. The schematic diagram of the co-sputtering process used to deposit our thin-film samples.

and $\mathrm{Cu}$, were first melted in a laboratory-scale arc-melting furnace with a high vacuum of $8 \times 10^{-4} \mathrm{~Pa}$ and $\mathrm{a} \mathrm{Ti}$ ingot to soak up the remaining oxygen. After that, the samples were cast into a $\mathrm{Cu}$ mold with the dimension of $5 \mathrm{~mm} \times 10 \mathrm{~mm} \times 50 \mathrm{~mm}$. For subsequent characterization, the as-cast samples were cut into a proper size for grinding and polishing.

Preparation of ribbon samples through vacuum melt spinning. We used metals with a purity level higher than $99.9 \%$ to prepare the ingots, which had the compositions of $(\mathrm{FeCrNi})_{2}(\mathrm{ZrCu})_{8,}(\mathrm{FeCrNi})_{15}(\mathrm{ZrCu})_{8.5}$, and $(\mathrm{FeCr}-$ $\mathrm{Ni})_{1}(\mathrm{ZrCu})_{9}$. The raw metals were melted and solidified to form the ingots in a laboratory-scale arc-melting furnace, which had a vacuum as high as $8 \times 10^{-4} \mathrm{~Pa}$ and contained a Ti ingot to soak up any residual oxygen in the vacuum. Then the ribbon samples were prepared via single roller melt spinning at a wheel surface velocity of $70 \mathrm{~m} / \mathrm{s}$ in a laboratory-scale induction-melting furnace with a high vacuum of $8 \times 10^{-4} \mathrm{~Pa}$.
Deposition of thin films through co-sputtering. As shown in Fig. 9, the cosputtering technique was utilized with two targets, one being FeCrNi and the other being $\mathrm{ZrCu}$. Both the $\mathrm{FeCrNi}$ and $\mathrm{ZrCu}$ are of equal-atomic ratio and with a purity level higher than $99.9 \%$. The FeCrNi target was sputtered on a direct-current source with a power of $200 \mathrm{~W}$, whereas $\mathrm{ZrCu}$ target was sputtered on a radio-frequency source with a power of $50 \mathrm{~W}$. Argon with a purity of $99.9 \%$ was applied as the protection gas with a pressure of 20 mtorr after pumping the furnance to $9 \times 10^{-6}$ torr. Eighteen pieces of wafers with a size of $5 \mathrm{~mm} \times 10 \mathrm{~mm}$ were arranged as a single line from the $\mathrm{FeCrNi}$ (left) side to the $\mathrm{ZrCu}$ (right) side. After a 40 min co-sputtering, a series of thin films with gradient components were prepared.

\section{DATA AVAILABILITY}

All data used in this manuscript are available from the authors on request.

\section{CODE AVAILABILITY}

All the codes used in this manuscript can be accessed via the following link https:// github.com/ZHOU-Ziqing/MLcode-for-HEAphase.

Received: 24 July 2019; Accepted: 2 December 2019;

Published online: 20 December 2019

\section{REFERENCES}

1. Yeh, J. W. et al. Nanostructured high-entropy alloys with multiple principal elements: novel alloy design concepts and outcomes. Adv. Eng. Mater. 6, 299-303 (2004). 
2. Miracle, D. B. \& Senkov, O. N. A critical review of high entropy alloys and related concepts. Acta Mater. 122, 448-511 (2017).

3. Zhang, Y. et al. Microstructures and properties of high-entropy alloys. Prog. Mater Sci. 61, 1-93 (2014)

4. Ye, Y. F., Wang, Q., Lu, J., Liu, C. T. \& Yang, Y. High-entropy alloy: challenges and prospects. Mater. Today 19, 349-362 (2016).

5. MacDonald, B. E. et al. Rerch. JOM 69, 2024-2031 (2017)

6. Mizutani, U. Hume-Rothery Rules for Structurally Complex Alloy Phases. (CRC Press: Boca Raton, 2016). 323-329.

7. Cantor, B., Chang, I. T. H., Knight, P. \& Vincent, A. J. B. Microstructural development in equiatomic multicomponent alloys. Mater. Sci. Eng. A 375-377, 213-218 (2004).

8. Zhang, Y., Zhou, Y. J., Lin, J. P., Chen, G. L. \& Liaw, P. K. Solid-solution phase formation rules for multi-component alloys. Adv. Eng. Mater. 10, 534-538 (2008).

9. Li, H. F. et al. In vitro and in vivo studies on biodegradable CaMgZnSrYb highentropy bulk metallic glass. Acta Biomater. 9, 8561-8573 (2013).

10. Cunliffe, A., Plummer, J., Figueroa, I. \& Todd, I. Glass formation in a high entropy alloy system by design. Intermetallics 23, 204-207 (2012).

11. Gao, X. Q. et al. High mixing entropy bulk metallic glasses. J. Non Cryst. Solids 357 3557-3560 (2011).

12. Chen, Y. Y. et al. Corrosion properties of a novel bulk Cu 0. 5 NiAlCoCrFeSi glassy alloy in $288^{\circ} \mathrm{C}$ high-purity water. Mater. Lett. 61, 2692-2696 (2007).

13. Guo, S., Ng, C., Lu, J. \& Liu, C. T. Effect of valence electron concentration on stability of fcc or bcc phase in high entropy alloys. J. Appl. Phys. 109, 103505 (2011).

14. Wang, Z., Huang, Y., Yang, Y., Wang, J. \& Liu, C. T. Atomic-size effect and solid solubility of multicomponent alloys. Scr. Mater. 94, 28-31 (2015).

15. Ye, Y. F., Liu, C. T. \& Yang, Y. A geometric model for intrinsic residual strain and phase stability in high entropy alloys. Acta Mater. 94, 152-161 (2015).

16. Zhang, Y., Yang, X. \& Liaw, P. K. Alloy design and properties optimization of highentropy alloys. JOM 64, 830-838 (2012).

17. Ye, Y. F., Wang, Q., Lu, J., Liu, C. T. \& Yang, Y. Design of high entropy alloys: a single-parameter thermodynamic rule. Scr. Mater. 104, 53-55 (2015).

18. Guo, S. \& Liu, C. T. Phase stability in high entropy alloys: Formation of solidsolution phase or amorphous phase. Prog. Nat. Sci. Mater. Int. 21, 433-446 (2011).

19. Yang, X. \& Zhang, Y. Prediction of high-entropy stabilized solid-solution in multicomponent alloys. Mater. Chem. Phys. 132, 233-238 (2012).

20. Guo, S., Hu, Q., Ng, C. \& Liu, C. T. More than entropy in high-entropy alloys: forming solid solutions or amorphous phase. Intermetallics 41, 96-103 (2013).

21. Rajan, K. Materials informatics: an introduction. Inform. Mater. Sci. Eng. DataDriven Discov. Accel. Exp. Appl. 8, 1-16 (2013).

22. Senkov, O. N., Miller, J. D., Miracle, D. B. \& Woodward, C. Accelerated exploration of multi-principal element alloys for structural applications. Calphad Comput. Coupling Phase Diagr. Thermochem. 50, 32-48 (2015).

23. Inoue, A. Stabilization of metallic supercooled liquid and bulk amorphous alloys. Acta Mater. 48, 279-306 (2000).

24. Ng, C., Guo, S., Luan, J., Shi, S. \& Liu, C. T. Entropy-driven phase stability and slow diffusion kinetics in an $\mathrm{Al} 0.5 \mathrm{CoCrCuFeNi}$ high entropy alloy. Intermetallics $\mathbf{3 1}$, 165-172 (2012)

25. He, Q. F., Ye, Y. F. \& Yang, Y. The configurational entropy of mixing of metastable random solid solution in complex multicomponent alloys. J. Appl. Phys. 120 154902 (2016).

26. He, Q. F., Ding, Z. Y., Ye, Y. F. \& Yang, Y. Design of high-entropy alloy: a perspective from nonideal mixing. JOM 69, 2092-2098 (2017).

27. An, S. et al. Common mechanism for controlling polymorph selection during crystallization in supercooled metallic liquids. Acta Mater. 161, 367-373 (2018).

28. Stillinger, F. H. A topographic view of supercooled liquids and glass formation Science 267, 1935-1939 (1995).

29. Debenedetti, P. G. \& Stillinger, F. H. Supercooled liquids and the glass transition. Nature 410, 259-267 (2001)

30. Wei, S., He, F. \& Tasan, C. C. Metastability in high-entropy alloys: a review. J. Mater. Res. 33, 2924-2937 (2018).

31. Takeuchi, A. \& Inoue, A. Classification of bulk metallic glasses by atomic size difference, heat of mixing and period of constituent elements and its application to characterization of the main alloying element. Mater. Trans. 46, 2817-2829 (2005).

32. Dong, Y., Lu, Y., Jiang, L., Wang, T. \& Li, T. Effects of electro-negativity on the stability of topologically close-packed phase in high entropy alloys. Intermetallics 52, 105-109 (2014)

33. Schmidt, J., Marques, M. R. G., Botti, S. \& Marques, M. A. L. Recent advances and applications of machine learning in solid-state materials science. npj Comput. Mater. 5, 83 (2019)

34. Bhadeshia, H. K. D. H., Dimitriu, R. C., Forsik, S., Pak, J. H. \& Ryu, J. H. Performance of neural networks in materials science. Mater. Sci. Technol. 25, 504-510 (2009).

35. Islam, N., Huang, W. \& Zhuang, H. L. Machine learning for phase selection in multi-principal element alloys. Comput. Mater. Sci. 150, 230-235 (2018).
36. Huang, W., Martin, P. \& Zhuang, H. L. Machine-learning phase prediction of highentropy alloys. Acta Mater. 169, 225-236 (2019).

37. Boser, E., Vapnik, N., Guyon, I. M. \& Laboratories, T. B. Training algorithm margin for optimal classifiers. Proceedings of the 5th Annual ACM Workshop on Computational Learning Theory. 144-152 (1992).

38. Yeh, J. W. Physical metallurgy of high-entropy alloys. JOM 67, 2254-2261 (2015).

39. Guo, S. Phase selection rules for cast high entropy alloys: an overview. Mater. Sci. Technol. 31, 1223-1230 (2015).

40. Butler, K. T. \& Daniel, W. Review Machine learning for molecular and materials science. Nature 559, 547-555 (2018).

41. Mizutani, U. Hume-Rothery rules for structurally complex alloy phases. MRS Bull. 37, 169-169 (2012)

42. Wang, Z., Guo, S. \& Liu, C. T. Phase selection in high-entropy alloys: from nonequilibrium to equilibrium. JOM 66, 1966-1972 (2014)

43. Lu, Z. \& Li, J. Correlation between average melting temperature and glass transition temperature in metallic glasses. Appl. Phys. Lett. 94, 2008-2010 (2009).

44. Turnbull, D. Under what conditions can a glass be formed? Contemp. Phys. 10 473-488 (1969).

45. Fukuhara, M., Takahashi, M., Kawazoe, Y. \& Inoue, A. Role of valence electrons for formation of glassy alloys. J. Alloy. Compd. 483, 623-626 (2009).

46. Takeuchi, T. et al. Free-energy analysis of the Zr-Ni-Al bulk metallic glass from the local atomic arrangements of the relevant crystals. Mater. Trans. 46, 2791-2798 (2005)

\section{ACKNOWLEDGEMENTS}

The research of Y.Y. is supported by City University of Hong Kong with the grant number 9610391 and by the Research Grants Council (RGC), the Hong Kong government, through the General Research Fund (GRF) with the project number CityU11213118 and CityU11209317.

\section{AUTHOR CONTRIBUTIONS}

Y.Y. supervised the project and conceived the idea. Z.Q.Z. collected and analyzed the data with the ANN algorithm, derived the sensitivity matrix, and carried out the experiments with the assistance of Q.F.H., D.Z.Y. and F.C.L. Y.J.Z. analyzed the data with the CNN and SVM algorithm. Z.Q.Z., Y.J.Z. and Y.Y. wrote the manuscript. All authors contributed to the discussion of the data.

\section{COMPETING INTERESTS}

The authors declare no competing interests.

\section{ADDITIONAL INFORMATION}

Supplementary information is available for this paper at https://doi.org/10.1038/ s41524-019-0265-1.

Correspondence and requests for materials should be addressed to Y.Y.

Reprints and permission information is available at http://www.nature.com/ reprints

Publisher's note Springer Nature remains neutral with regard to jurisdictional claims in published maps and institutional affiliations.

Open Access This article is licensed under a Creative Commons Attribution 4.0 International License, which permits use, sharing, adaptation, distribution and reproduction in any medium or format, as long as you give appropriate credit to the original author(s) and the source, provide a link to the Creative Commons license, and indicate if changes were made. The images or other third party material in this article are included in the article's Creative Commons license, unless indicated otherwise in a credit line to the material. If material is not included in the article's Creative Commons license and your intended use is not permitted by statutory regulation or exceeds the permitted use, you will need to obtain permission directly from the copyright holder. To view a copy of this license, visit http://creativecommons. org/licenses/by/4.0/.

(c) The Author(s) 2019 